\title{
Usability Guidelines for an Online Image Database
}

\author{
Ruth Chang, Donald F. Bliss II and Anne R. Altemus
}

\begin{abstract}
There are currently no existing guidelines for optimizing user experience in online image databases. Here we use established usability design principles to develop a set of 50 recommendations for improving database organization, navigability, and search functionality. We conduct a resource audit of commercial and non-profit image galleries and evaluate them upon our criteria. Our findings are summarized within a basic wireframe to be used as reference for prospective image databases in the future.
\end{abstract}

OPEN ACCESS

\section{Keywords}

Image database, user experience, usability, searching, content management

\section{Introduction}

As the size of a digital image collection increases, so then does the need for effective organization of the images within. Online image databases have been adopted by both commercial and non-profit institutions as a platform for users to access images through browsing or search functionality. While a sizeable number of studies are dedicated to the development of database management systems, less attention has been focused on front end user interface (UI) design. Much of the existing research examining user interactivity with databases is either outdated (Conner 1993; Oren 1995; Szabo 1999), or else preoccupied with using user-generated data to refine search results. (Catarci 2000; Jagadish 2007) Thus, there are currently no existing design guidelines in place for optimizing user experience in online image databases.

The key objective of any successful design is to maximize usability while minimizing negative side-effects. (Bevan 2009) Although the concept of usability is broad, as Rubin and Chisnell (2008) observe, usability can be summarized by the absence of frustration when using a product. Good usability begins with the method through which information is presented and extends to encompass the purpose and constraints of the design as a whole. (Dix 2009) In the context of an image database, this means that the user is able to effectively locate their desired image, in an efficient amount of time, and in a satisfactory manner. (Bevan 2009) Fortunately, there is a wealth of existing literature detailing principles and standards for online usability. These include wide-ranging design axioms: striving for consistency, offering informative feedback, and reducing short-term memory load on the user (Shneiderman 2010); as well as more specific examples looking at individual UI elements, such as those documented in Research-Based Web Design and Usability Guidelines by the US Department of Health and Human Services.

Here, we apply research-based usability design principles to develop a set of parameters relevant to online image databases. We discuss the importance and impact of each of these criteria in streamlining user experience. Examples of commercial and non-profit online image database resources are then quantitatively and qualitatively evaluated upon these parameters to determine which usability standards are ubiquitous, and which are atypically used. Our resulting conclusions are encapsulated within a basic wireframe. Taken together, these findings serve as a future guideline for image database usability.

\section{Methods}

Drawing from government-standard usability protocols, we determined three main categories for evaluation criteria: Content Management Strategy, UI Design, and Services. These categories were further divided into subcategories according to relevance and contain 50 individual criteria in total. We conducted a resource audit of 12 online image databases, and qualitatively measured them upon the parameters established. The resources were selected for diversity in traffic (based on average number of visits per month) and profitability (commercial vs. non-profit), with a preference for scientific image databases. Website traffic was determined through SimilarWeb, an online website traffic statistics resource. The non-profit databases assessed included, in order of decreasing web traffic: Library of Congress Prints \& Photographs Online, The New York Public Library (NYPL) Digital Collections, Open-i Biomedical Image Search Engine, National Library of Medicine (NLM) Digital Collections, 
Centers for Disease Control and Prevention Public Health Image Library (CDC-PHIL), National Cancer Institute (NCI) Visuals Online, and the Cell Image Library. The commercial databases assessed included, also in order of decreasing web traffic: Shutterstock, iStock, Science Photo Library, Science Source, and A.D.A.M. Images.

The design survey of each resource involved navigating through the database and user-testing each parameter individually, and the results can be found in Additional Data. Resources were given a filled circle if the criterion was fulfilled successfully, a half circle if the criterion was fulfilled partially, an empty circle if the criterion was not fulfilled, and no marking if the criterion was not assessed or not applicable. The data was then inputted into a survey tally matrix, where it was then analyzed and compared against other image databases to assess the comprehensive usability of each resource, as well as the overall performance of the resources regarding each parameter. Our final wireframe and recommendations are based upon the results.

\section{Results}

\section{Content Management Strategy}

Arguably the most important aspect of a database is how raw data can be organized in a way that is meaningful and accessible. Poor content management undermines the very purpose of a database. The way an inventory is structured will ultimately determine the findability of the content. There are three main aspects to a content management strategy that helps to refine the user search methodology: organization schemes, search refinement, and metadata. (Table 1)

\section{Organization Schemes}

Organization schemes refer to the categorical relationships between individual images in the inventory. Schemes can be divided into exact and subjective. Exact organization schemes split content into mutually exclusive sections. Although such organization schemes are unambiguous and objective in how they organize information, the user is obligated to have preexisting knowledge of their target image. (Rosenfeld and Morville 2002)

Alphabetical: Such an organization scheme is only applicable to languages with alphabets. It should be made clear what exactly is being sorted alphabetically (i.e. Title, Artist, Publisher, etc.) This type of organization is more applicable to reference databases such as libraries where metadata is prioritized. Few of the commercial databases analyzed offered this option.

Chronological: Organizing content by date similarly requires specification on what is being sorted chronologically (i.e. Date created, Date published, Time period/Era, etc.). Oftentimes chronological schemes will be presented as "Newest" or "Most Recent", an organization scheme which encourages frequent visitation of the website.

License: Somewhat unique to media databases, sorting by license allows users to immediately narrow down their search to images that fit their usage requirements. This is particularly useful for users searching for visual assets that they intend to redistribute.

Table 1. Content Management Strategy

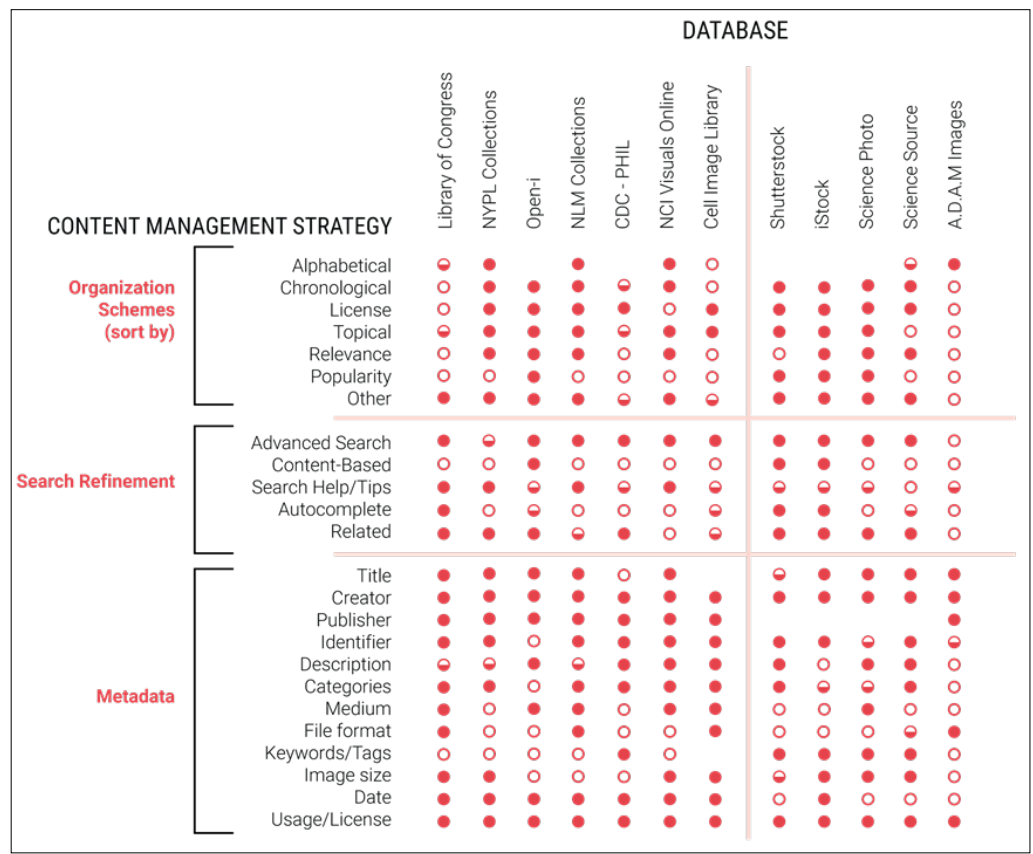


Subjective organization schemes, on the other hand, are not as precise and are more difficult to design. However, they are more user-friendly and intellectually meaningful than exact schemes, simply because sometimes users do not know what they are looking for. (Rosenfeld and Morville 2002) Subjective organization schemes form an essential part of the browsing process when the user lacks a specific end goal in mind, or has only cursory knowledge of the target of their searches. (Usability.gov 2016)

Topical: Organizing information by topic is perhaps the most useful categorization feature provided by online databases, and often takes the form of tags or keywords. However, there is a danger inherent to using a single word to describe a picture (which is allegedly worth $10^{3}$ as many): a tremendous proportion of searchable content becomes dependent on the language and representation of a one-word label. (Morville and Sullenger 2010) Topical organization should thus always be accompanied by the option of other organization scheme to filter search results if necessary.

Relevance: Sorting by relevance is frequently seen as well, but the word itself is ambiguous. Relevance is a generic term that refers to an algorithm, which ranks search results in an order that would most likely interest the user. As different databases have different ranking needs, so changes the type of algorithm used. (Lextek 2000)

Popularity: This method of sorting also varies by algorithm. It usually indicates the number of views or downloads an image has. As a marketing tool, it could also be used by a database to promote certain images, effectively taking advantage of a user's inclination to be influenced by group consensus.

This is by no means an exhaustive list on schemes to organize content. Other schemes that our resource audit encountered included sorting by Collection, by Format, by Genre, by Image Type, by Image Orientation (vertical or horizontal), by Price, and by Quality. The last three were observed exclusively among commercial galleries.

\section{Search Refinement}

Search refinement helps to limit the scope of the search process, and user search experience tends to benefit from search refinement options, particularly for larger collections of searchable items. (HHS.gov 2016). However, search refinement options should be applied conservatively and take into account the target audience, as casual users may easily become overwhelmed by the different search options available. It would thus be more constructive to offer search refinement options only after the initial search turns up too many results. (Krug 2005)

Advanced Search: Although most users work only with simple searches, users with web expertise rely significantly on advanced search functionality such as Boolean operators when compared to web novices, especially on information-rich sites. (Hölscher 2000). Casual users who are not familiar with query formatting may be encouraged to conduct more thorough searches through clearly labeled features such as Include/Exclude, Match any/all/exact, etc.

Content-based: A sizeable area of research unto itself, content-based image retrieval (CBIR) allows users to find an image based on its visual qualities, such as color, texture, and shape, rather than content. (Rui et al. 2009) This has powerful implications in the field of medical imaging; CBIR could serve as a diagnostic aid, not only in retrieving patients with similar diagnoses, but also in identifying patients with visually similar but different diagnoses. (Müller et al. 2004) Some examples of general CBIR engines include Google Reverse Image Search and TinEye.

Search Help/Tips: The availability of semantic and syntactic search hints directly improves user effectiveness, efficiency, and confidence when conducting searches. (Bandos and Resnick 2004) A link to these hints should be located near the Search Field if possible, or turn up automatically if the user is unable to find any results.

Autocomplete: Also called predictive search, the autocomplete feature allows users to accurately and quickly specify their search query from a curated list, thus reducing the cognitive load on their working memory. Autocomplete suggestions might be ordered alphabetically or based on popularity. (Laubheimer 2016)

Related: Providing the users with examples of other images that may interest them encourages browsing and may save users from having to refine their search query. (van Welie 2008) 


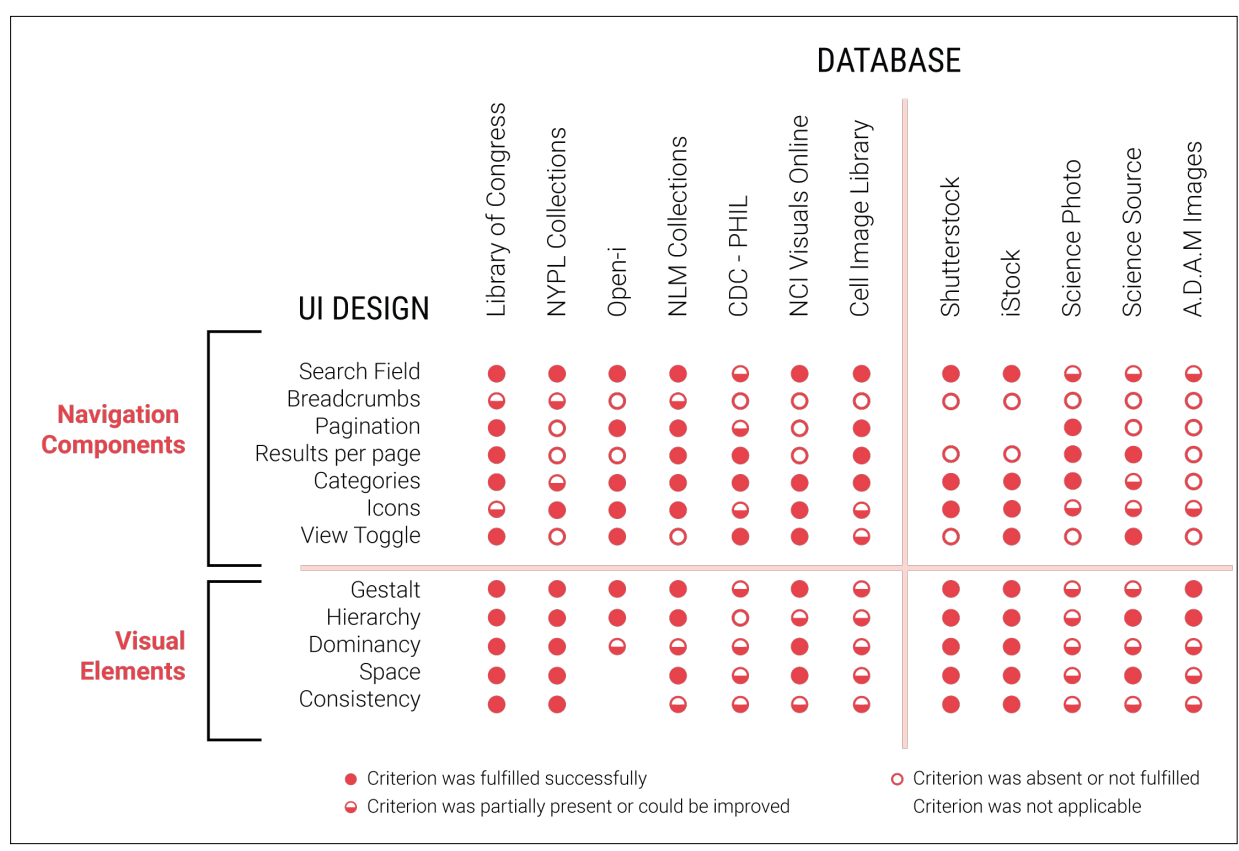

Table 2. User Interface (UI) Guidelines

\section{Metadata}

Metadata, or "structured data about data", is used to document, manage, and retrieve resources within collections. Content management systems track the descriptive metadata encoded within inventory objects to conduct search functionality, so it follows that metadata plays a huge role in how users locate information. Different metadata schemes have been adopted as standards throughout various disciplines. Their role is to define a limited number of elements to describe a resource, and the naming, meaning, and rules for the use of each element. (Government of Canada 2016) Some familiar metadata schemes used in image databases may include Dublin Core for general collections, Metadata Object Description Schema (MODS) for librarianship, and Visual Resources Association (VRA) Core for visual culture. In each instance, a metadata scheme provides controlled parameters to support consistency in searching and browsing.

Inventories vary drastically in what they consider to be important, and thus in which metadata scheme they use, and subsequently how much data they provide. Predictably, reference libraries adhere to structured metadata standards for archival purposes, and share the most information about their collections. Commercial stock image galleries provide very little metadata, instead preferring to use user-generated Keywords or Tags to track their inventory. The list in Table 1 covers some examples of appropriate metadata elements that are commonly used in image databases across metadata schemes. (DCMI 2016; Usability.gov2016)

\section{User Interface Design}

The objective of user interface (UI) design is to ultimately guide users toward what they are looking for. There are many existing guidelines and principles in place explaining how to overcome the usability issues that plague bad interface design. Cluttered displays, inconsistent navigation components, and poor use of visual salience are just some examples of factors, which contribute to user stress and frustration. (Shneiderman 2010) In this section, we evaluate our online image databases on two categories: usage of navigation components and usage of visual elements. (Table 2) While these UI guidelines may not be one-size-fits-all, they nonetheless have been supported by research studies and have become a reliable staple of web design.

\section{Navigational Components}

A well-designed navigation system provides context and flexibility to a search engine. Users have grown accustomed to seeing certain navigation elements repeated throughout the websites they visit, and they have built up a mental model of how navigability should work. This primes them to expect familiar components in their UI, leading to confusion when these components are not present. (Nielsen 2010) The following is a list of frequently used navigation components applicable to image databases:

Search Field: The keystone of any database, a search field allows users to enter a query and ideally returns useful results. The search field should provide space for at least 20 characters, to allow for longer search queries. (van Welie 2008) Include no more than a single search box, which should be accessible regardless of where the user is within the database. (Nielsen 2003)

Breadcrumbs: Breadcrumbs are a textual representation of site structure. Much like a "You are here" arrow, breadcrumbs help users identify their location within a website, orienting them within the site hierarchy and improving navigation efficiency. (Hull 2004) Each element in the breadcrumb should be a link to allow for backpedaling, but the number of clickable breadcrumbs should not exceed four. (HHS.gov) 
Pagination: Used widely by search engines, pagination divides content into separate chunks for easier readability. Pagination is preferred by users over continuous scrolling, which limits search efficiency and is perceived as being cluttered (Bernard et al., 2002). Page numbers should be flanked on each side by First, Previous, and Next, Last links. If the number of available pages exceeds around 10, additional pages between the first and last few should be represented by three ellipses. (Lennartz 2007)

Results per Page: Allowing users to choose how many results are returned per page of pagination empowers them to customize their search preferences. Options should be significantly different (i.e. $10,50,100$ ) for the feature to be useful. The upper limit of results shown should be around 100 items, as offering a "View All" option may impact response time and be detrimental to user experience. (Nielsen 2013)

Categories: A necessary feature of browsing, sorting images by category allows users who do not know exactly what they are looking for to sift through related pictures until they find what they want. (Usability.gov) Categories are often arranged in a hierarchy, with more specific subcategories beneath.

Icons: Although icons run the risk of having their intended meaning misconstrued, there are certain icons that have been used frequently enough that they have become standardized symbols. These include a magnifying glass for zooming or searching, an arrow pointing down for download, house for the home page, etc. When used appropriately, icons can decrease textual clutter, allowing for fast recognition. (Rosenfeld and Morville 2002) Icons should be findable, recognizable, informative, and attractive. (Bedford 2016)

View Toggle: Some image collections allow users to choose how they view the images, whether through thumbnails, enlarged images, or a slideshow. This feature helps give users a sense of control, and is useful for those who may have poor vision, or for users who are trying to distinguish between several visually similar images.

\section{Visual Elements}

When the average user spends less than a minute on a webpage (Nielsen 2011), there is precious little time to present a UI layout that is both aesthetically pleasing and easily comprehensible. Users focus only on words or features related to their goal. This is where effective use of visual elements comes into play: by using salience cues to organize information by importance, users are able to obtain a clear visual roadmap for the database. (Krug 2005) A successful visual design does not detract from the content of the UI, but instead enhances user experience and decreases cognitive overload.

Gestalt: Gestalt is the idea that an organized whole appears greater than the sum of its parts. In design, gestalt is a clear, overall feel for a design that promotes a sense of unity and congruency. (Usability.gov 2016) This criterion evaluates whether a resource successfully conveys a cohesive brand and purpose.

Dominancy: Essential for the user to locate themselves, dominancy provides context within the greater whole of the interface, facilitating navigation. Dominance is often established through contrasting based on size, color, position, or shape. (Usability.gov 2016) For example, the title and main navigation of the page should always be large and at the top of an interface. In an image database, where searching is the main functionality, the search bar should be clear and obvious.

Hierarchy: Hierarchy is similar to dominancy in that it serves as a crucial landmark for users. If dominancy helps users locate themselves on a map, hierarchy is the organization that allows the user to explore the rest of the map in a rational and familiar manner. Links that are related logically are visually grouped together, and content is nested within headings or categories to create associations. (Krug 2005) While hierarchal navigation should present information in order of importance, hypertext allows users to move laterally among webpages and therefore bypass the limitations of hierarchy. While useful, this increased flexibility must be used carefully, lest users become loss in an overly complex website. (Rosenfeld and Morville 2002)

Space: When a UI is crowded and high in complexity, individual elements start competing for user attention. Space plays a key role in reducing visual noise and cognitive load. Incorporating space improves readability and places emphasis on individual elements. (Usability.gov 2016)

Consistency: Although there may be reluctance among designers to follow design conventions, it is undoubtedly advantageous to do so. Following conventions allows for predictability, and predictability lowers cognitive load on users, consequently increasing search efficiency. (Krug 2005) Websites must be consistent not just with other websites, but also within itself as well: for instance, a navigation component should be located in the same place from page to page (also known as persistent or global navigation). 


\section{Services}

The goal of an image database determines some of the services it offers. A library collection might offer an Ask a Librarian chat box for prompt help with locating reference material, while a commercial website might sell memberships for access to high resolution photos. In any case, while the goals of the image databases may differ, the primary goal of the user does not change: the user needs to find one or multiple images to use. Here we have compiled a list of services that are helpful for the user to achieve their goal. The online resources were evaluated upon these criteria. (Table 3)
High Resolution: High-resolution versions of images are available from commercial galleries, and sometimes offered freely by non-profit galleries, depending on the copyright.

Download Help: A Help section focusing on Downloads would benefit casual users who are unfamiliar with how to save images to their computer, or else do not know which file format is suitable for their needs.

Print Options: Also directed at casual users, a printer icon is shown near the image that opens the printer dialog when clicked.

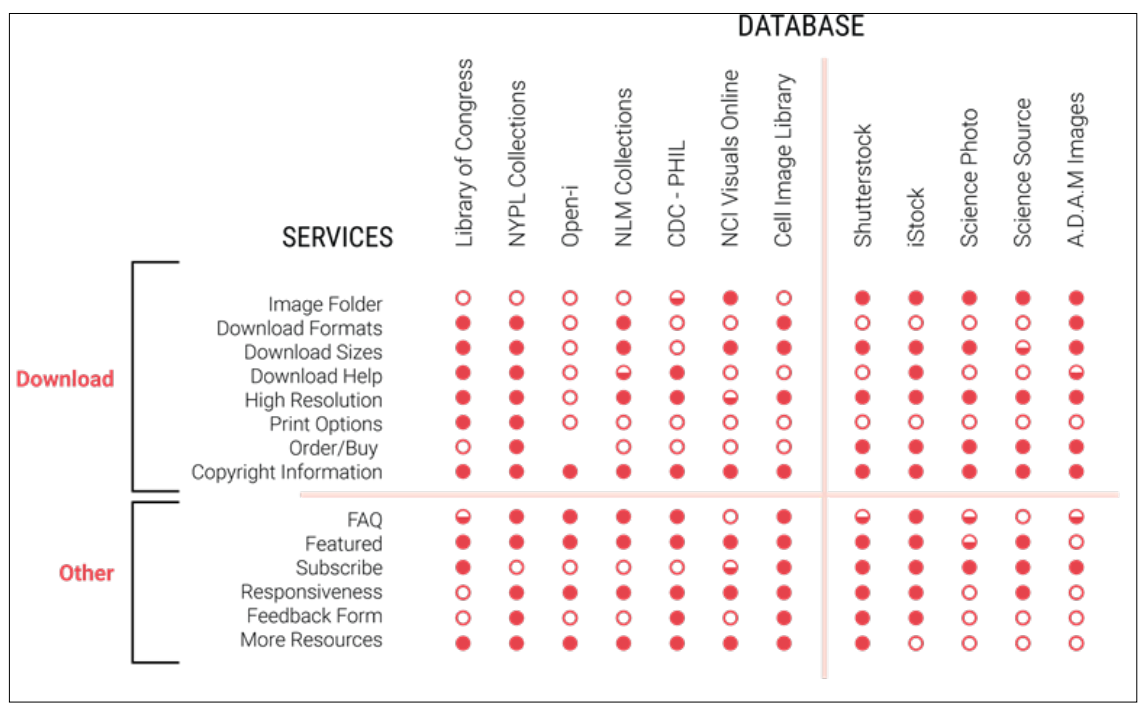

Order/Buy: Image databases may allow users to purchase high resolution and vector files of images, or physical prints. While this option is the main purpose of commercial websites, it is rarely seen among non-profits.

Copyright Information: Users will want to know the licensing of the image, and whether they are allowed to modify, use, or publish the image in any way.

Table 3. Services

\section{Download}

To save an image onto their computer, users must first download the image. The download process could be facilitated with a variety of services and options.

Image Folder: Also known as "Lightboxes", image folders allows users to select and store multiple images on a separate webpage for later viewing as they browse through the collection.

Download Formats: Some image databases provide images for download in different file formats. While the most common format is JPEG for lower resolution pictures and TIFF for high resolution, there are some resources that offer GIF, EPS, or PNG download options as well.

Download Sizes: Images may be available for download in different sizes. On commercial websites, price increases accordingly with image size.

\section{Other}

FAQ: Frequently Asked Questions is the go-to page when a user does not understand a concept. FAQ sections should be easily accessible, and actually contain a list of relevant questions (and not just self-promoting summaries phrased in question form).

Featured: Featured sections are a curated collection of images updated intermittently to add browsing interest and encourage users to revisit the site.

Subscribe: Subscription functions are useful if a user would like to receive updates or news. Similar to a Featured section, subscription is a method of advertising that encourages repeat visitors.

Responsiveness: Users access online resources through a variety of screen sizes, and responsiveness is becoming a necessity as mobile users overtake desktop users in number. 
Feedback Form: A good way to improve usability is to solicit feedback from the users themselves. Although pop-up dialogs tend to aggravate users, there are subtler ways to allow users the chance to provide their opinion about how the image database is run.

More Resources: Oftentimes image galleries will offer additional help or information regarding selected images. Reference databases may refer the user to the image's location within a greater collection, while commercial databases may provide built-in image editing features.

\section{Discussion}

\section{Overview of existing conventions}

We set out to determine standard guidelines for online image database using evidence-based research, and established 50 individual criteria as a result. Our resource audit identifies the most common characteristics and UI components shared by currently available image databases. These results outline an organization scheme that is predictable and familiar to the user, which is critical to supporting the user's existing mental models and increasing usability. (Nielsen, 2010) Our findings are summarized with a wireframe in Figure 1 and 2.

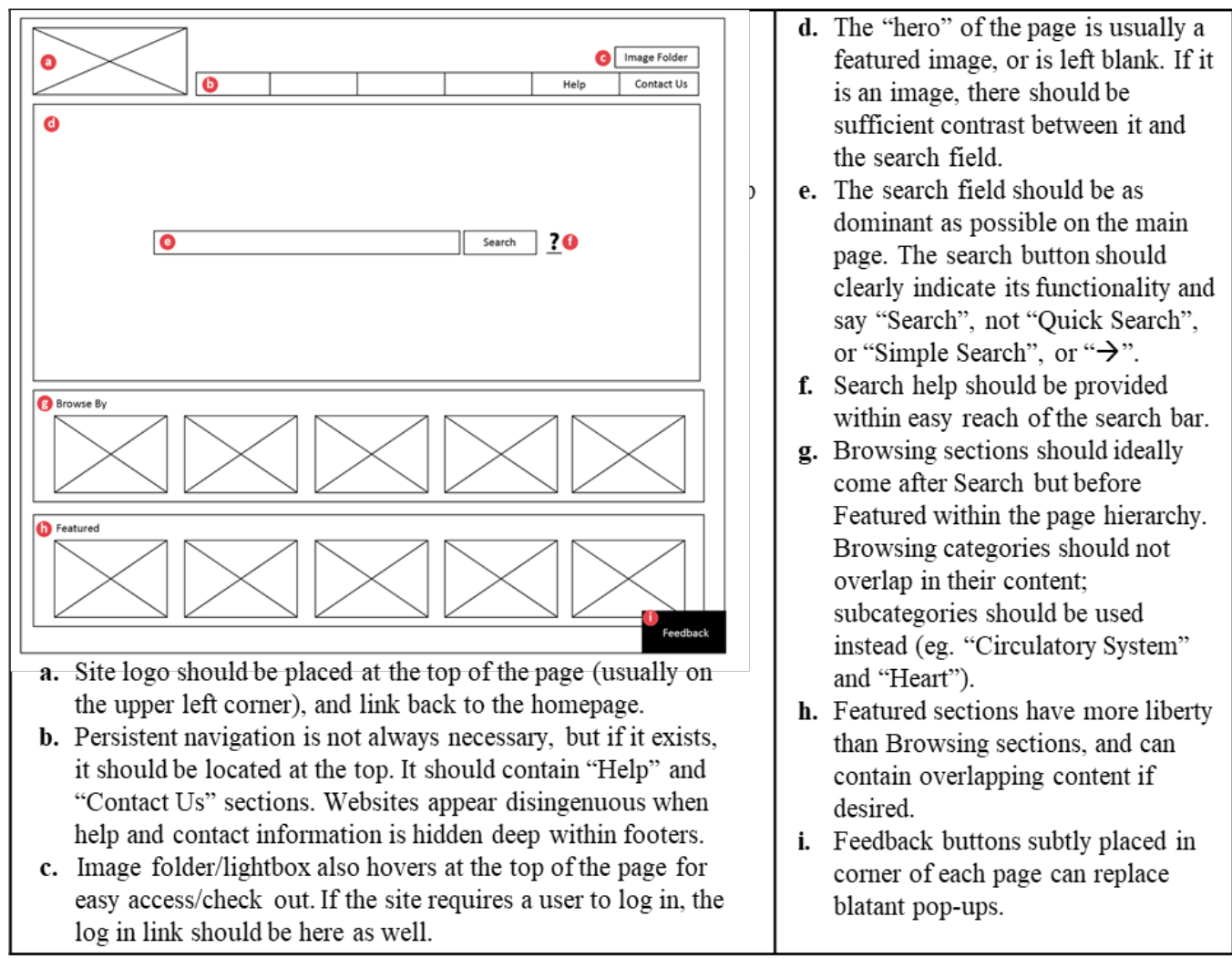

Figure 1. Homepage wireframe
We were also able to single out elements which, despite being frequently encountered online, seem to be less prevalent among image databases. An example of this is breadcrumb navigation, which was used only by 3 out of the 12 resources. A reason for this may be that image databases tend to have flat rather than deep content hierarchies; that is, images are discoverable in fewer clicks, without being buried under multiple sublevels (Whitenton 2013). Nonetheless, image databases with multiple categories and subcategories may still benefit from breadcrumb navigation to allow users to backtrack easily.

Several recurring usability hurdles presented themselves during our resource audit. First, although autocomplete is a welcome feature, autocomplete suggestions should be appropriately curated. (Table 1) Some databases had repetitive or misspelt suggestions that were obviously based upon previous user input. Second, search fields were inconspicuous and obscured in some of the resources we looked at. (Table 2) Some resources misleadingly included two separate search fields (presumably for different areas of the site). As the main function of a database is searching, the search field should always have dominance in an UI. (Figure 1e) Third, search tips were often buried deep within several layers of links only accessible from the footer of the page.

(Table 1) A more intuitive solution is to provide a link or icon to search help beside the search bar itself. (Figure 1f) Finally, many databases limited their organization schemes to browsing only. (Table 1) This meant that, upon receiving a list of search results, users could not order it in the way they could a list of browsing results. An effort should be made to provide consistency between sorting functions for searching and browsing. (Figure 2a,c)

There were other unexpected design issues uncovered during the resource audit that were less relevant to the searching process, but nevertheless hindered the user experience. For instance, social media icons such as the Facebook " $\mathrm{f}$ ", 
Twitter bird, and Instagram camera are now ubiquitous across the web. However, there was inconsistency regarding the purpose of these icons; upon clicking on them, some websites directed you to their respective social media pages, while others instead connected you to your own social media platform as an invitation to "share" or otherwise promote their website. Similarly, although the most popular icons (ie. for zooming in/out, for downloading) were largely consistent, some of the more infrequently encountered icons (ie. for similar images, for lightbox) served to confuse rather than clarify. In such cases, icon usage should either be reconsidered, or instead yield a tooltip upon hovering that states the intended purpose of the icon.

(Table 2)

\section{Comparing commercial and non-profit image databases}

There are distinct differences between the design of commercial and non-commercial databases outside of the ability to pay for high resolution images. Evidently, libraries and other non-profit institutions offer far more metadata information than their commercial counterparts, but seldom

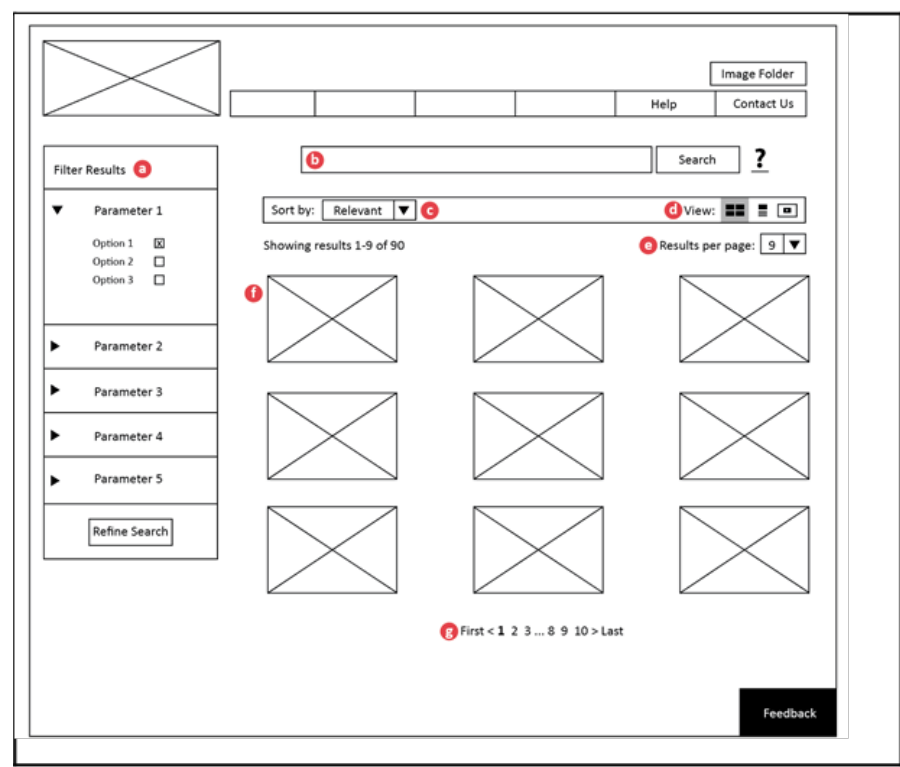

Figure 2. Search wireframe

used keywords (or tags) to organize their content. Commercial databases, on the other hand, rely almost entirely on tags to locate images. Non-profit galleries are also more inclined to offer advanced search functionality and a variety of download formats, and to solicit user feedback. They were less likely to sort search results by popularity or copyright license (Table 1).

There are also dissimilarities between commercial image databases themselves. Large-scale, general commercial databases (ie. Shutterstock and iStock, 60 million and 12 million visitors/month respectively) model their home pages after Google Image Search, displaying a prominent and central search field surrounded by negative space, a stylization only shared by Open-i. Also shared with Open-i is their contentbased search functionality, allowing the user to find images by uploaded images or by color. These commercial databases have dispensed with navigable pagination entirely due to the sheer volume of images they have: a search would often turn up thousands of pages in results. As such, the "Results per page" criterion was deemed not applicable for these resources. Science-centric commercial image databases experience significantly less traffic (9000 to 200000 /month), with visitor numbers comparable to those of the non-profit organizations (with the exception of the Library of Congress). Interestingly, while these science image databases were slightly more generous with navigation components than the larger commercial databases, they often lacked adequate search refinement options and organization schemes (Table 1). Especially for the smaller science databases, this penalizes users who do not know exactly what they are searching for. This lapse in usability may be due to the precarious assumption that users of science image databases already have prerequisite knowledge about the image they are looking for. Or, it may simply be that smaller databases have less funding to conduct usability tests.

\section{Accessibility}

e. Results per page should be aligned with the number of total results.

f. Results themselves should be evenly spaced and may include identifying information below them or upon mouseover.

g. Pagination should be below the results.

Although we did not
include accessibility
within our evaluation, it
is worth stressing that
accessibility is an essential aspect of user-centered design. Accessibility standards ensure that people affected by physical disabilities, such as impaired vision, hearing, or movement, receive a comparable experience and are able to accomplish their goals when visiting a web site online. This includes providing text equivalents for non-text elements, and ensuring that colorbased information is also conveyable without color (Section508.gov, 2016). Optimizing product usability for people with disabilities oftentimes benefits those without disabilities as well. For instance, adjusting text contrast so that it is more readable for individuals with poor vision also assists 
those in poor lighting conditions (Rubin and Chisnell, 2008). There are many resources and best practices available regarding accessibility guidelines. For more information, visit https://www.section508.gov/

\section{Concluding Remarks}

We present these design principles as general recommendations to guide the creation of any prospective online image database. These standards are simply suggestions and are not laws, as the end goal of each individual image database inevitably differs. Nonetheless, we believe our findings will help improve the usability, organization, and navigability of future digital galleries.

\section{Acknowledgements}

This project was supported by the Intramural Research Program of the National Institutes of Health, National Library of Medicine.

\section{References}

Bandos, J. and Resnick, M. 2004. The effects of semantic and syntactic instruction on user performance and satisfaction in search user interface design. Proceedings of the Human Factors and Ergonomics Society Annual Meeting.

Bedford, A. 2016. Usability Testing of Icons. Nielsen Norman Group. https://www.nngroup.com/articles/icon-testing/

Bernard, M., Baker, R. and Fernandez, M. 2002. Paging vs. Scrolling: Looking for the Best Way to Present Search Results. Usability News. http://usabilitynews.org/paging-vsscrolling-looking-for-the-best-way-to-present-search-results/

Bevan, N. 2009. Usability. In Encyclopedia of Database Systems, 3247-3251. Springer.

Catarci, T. 2000. What happened when database researchers met usability? Information Systems 25(3): 177-212.

Conner, H.K., Petersen, D.G., Wang, J.S. and Wood, R.B. 1993. User interface for a relational database using a task object for defining search queries in response to a profile object, which describes user proficiency. Google Patents.
Dix, A. 2009. Human-Computer Interaction. In: Liu L., Özsu M.T. (eds) Encyclopedia of Database Systems. Boston: Springer.

Dublin Core Metadata Initiative (DCMI). 2016. "DCMI Metadata terms." http://www.dublincore.org/documents/dcmiterms/

Government of Canada. 2016. "Metadata (Data Structure Standards).” http://canada.pch.gc.ca/eng/1443536696557

Hölscher, C. and Strube, G. 2000. Web search behavior of Internet experts and newbies. Computer Networks 33(1): 337-346.

Hull, S.S. 2004. Influence of training and exposure on the usage of breadcrumb navigation. Usability News, 6.1

Jagadish, H.V., Chapman, A., Elkiss, A., Jayapandian, M., Li, Y., Nandi, A., and Yu, C. 2007. Making database systems usable. Proceedings of the 2007 ACM SIGMOD international conference on Management of data.

Krug, S. 2005. Don't make me think: A common sense approach to web usability. Berkeley: New Riders.

Laubheimer, P. 2016. 7 Ways to Improve Your Website's or Intranet's Built-In Search Engine. Nielsen Norman Group. https://www.nngroup.com/articles/internal-website-search/

Lennartz, S. 2007. Pagination Gallery: Examples and Good Practices. Smashing Magazine.

https:/www.smashingmagazine.com/2007/11/paginationgallery-examples-and-good-practices/\#good-practices-ofpagination-design 7-aspects-according-to-faruk-ates

Lextek. 2000. "Relevancy Ranking."

http://www.lextek.com/manuals/onix/ranking.html

Morville, P., and Sullenger, P. 2010. Ambient findability: libraries, serials, and the internet of things. The Serials Librarian 58(1-4): 33-38.

Müller, H., Michoux, N., Bandon, D. and Geissbuhler, A. 2004. A review of content-based image retrieval systems in medical applications - clinical benefits and future directions. International Journal of Medical Informatics 73 (1): 1-23.

Nielsen, J. 2003. Employee Directory Search: Resolving Conflicting Usability Guidelines. Nielsen Normal Group. https://www.nngroup.com/articles/employee-directory-search/ 
Nielsen, J. 2011. How Long Do Users Stay on Web Pages? Nielsen Norman Group.

https://www.nngroup.com/articles/how-long-do-users-stay-onweb-pages/

Nielsen, J. 2010. Mental Models. Nielsen Norman Group. https://www.nngroup.com/articles/mental-models/

Nielsen, J. 2015. Users' Pagination Preferences and 'View All'. Nielsen Normal Group. https:/www.nngroup.com/articles/item-list-view-all/

Oren, T.R., Kreitman, K.M. and Salomon, G.B. 1995. User interface system and method for traversing a database. Google Patents.

Rosenfeld, Louis, and Morville, Peter. 2002. Information Architecture for the World Wide Web, 2nd Edition. Designing Large-Scale Web Sites. Boston: O’Reilly Media, Inc.

Rubin, Jeffrey, and Chisnell, Dana. 2008. Handbook of usability testing. Indianapolis: Wiley Publishing, Inc.

Rui, Y., Huang, T.S. and Chang, S. 1999. Image retrieval: Current techniques, promising directions, and open issues. Journal of Visual Communication and Image Representation 10(1):39-62.

Section508.gov. 2016. GSA Government-wide Section508 Accessibility Program. https://www.section508.gov/

Shneiderman, B. 2010. Designing the user interface: strategies for effective human-computer interaction. Pearson Education, India.

Szabo, A.J. 1999. Graphic user interface for database system. Google Patents.

US Department of Health and Human Services (HHS.gov). 2016. HHS Web Standards and Usability Guidelines. http://webstandards.hhs.gov/

US Department of Health and Human Services (usability.gov). 2016. "Research-Based Web Design and Usability Guidelines." http://www.usability.gov/pdfs/guidelines.html.

van Welie, M. 2008. Search Results. Patterns in Interaction Design http://www.welie.com/patterns/showPattern.php?patternID $=$ se $\underline{\text { arch-results }}$
Whitenton, K. 2013. Flat vs. Deep Website Hierarchies. Nielsen Norman Group.

https:/www.nngroup.com/articles/flat-vs-deep-hierarchy/

\section{Authors}

Ruth Chang, B.Sc., M.Sc. BMC is a graduate of the Master of Science in Biomedical Communications program at the University of Toronto. ruth.cj.chang@gmail.com

Donald F. Bliss II, M.A. works in the Office of Communication and Public Liaison at the National Library of Medicine, NIH, and is an Assistant Professor in the Department of Art As Applied to Medicine at the Johns Hopkins University School of Medicine.

blissd@mail.nih.gov

Anne R. Altemus, M.A., FAMI is a Senior Visual Information Specialist in the Office of the Director, Office of Communications and Public Liaison, National Library of Medicine, NIH, and Assistant Professor in the Department of Art As Applied to Medicine at the Johns Hopkins University School of Medicine. aaltemus@mail.nih.gov 